\title{
PROCEDIMENTOS PARA CONDUÇÃO DE TESTES DE VIGOR BASEADOS NA TOLERÂNCIA AO ESTRESSE TÉRMICO EM SEMENTES DE PEPINO ${ }^{1}$
}

\author{
LIANA BAPTISTA DE LIMA², JÚLIO MARCOS-FILHO³
}

\begin{abstract}
RESUMO - O presente trabalho foi realizado com o objetivo de identificar métodos, baseados na tolerância das sementes ao estresse térmico, eficientes para a avaliação do potencial fisiológico das sementes de pepino. O trabalho foi desenvolvido em três épocas, utilizando-se lotes de sementes dos cultivares Safira e Jóia, abrangendo testes de germinação, emergência de plântulas, envelhecimento acelerado e deterioração controlada. Concluiu-se que o teste de deterioração controlada (com ajuste do teor de água para $24 \%$, período de $48 \mathrm{~h}$ a $45^{\circ} \mathrm{C}$ ) e de envelhecimento acelerado tradicional e com solução saturada de cloreto do sódio (a $41^{\circ} \mathrm{C}$ por $96 \mathrm{~h}$ ) é sensível para a avaliação do potencial fisiológico de sementes de pepino.
\end{abstract}

Termos para indexação: análise de sementes, deterioração controlada, envelhecimento acelerado, Cucumber sativus.

\section{PROCEDURES FOR VIGOR TESTS BASED ON THE THERMAL STRESS TOLERANCE OF CUCUMBER SEEDS}

\begin{abstract}
The objective of this research was to identify reliable methods based on the thermal stress tolerance of cucumber seeds, which would be efficient for evaluating their physiological potential. The study was conducted in three experimental phases using seed lots of Safira and Joia cultivars and included procedures for the assessment of seed physiological potential from germination, field emergence, controlled deterioration and accelerated ageing tests. Results indicated that the controlled deterioration $\left(24 \%\right.$ water content/ $\left.48 \mathrm{~h} / 45^{\circ} \mathrm{C}\right)$, and the traditional and saturated salt accelerated ageing $\left(41^{\circ} \mathrm{C}\right.$ for $\left.96 \mathrm{~h}\right)$ tests are sensitive to differences in the physiological quality of cucumber seed lots.
\end{abstract}

Index terms: seeds analysis, controlled deterioration, accelerated ageing, Cucumber sativus.

${ }^{1}$ Submetido em. 12/02/2010. Aceito para publicação em 31/05/2010. Parte de tese de doutorado do primeiro autor realizado com bolsa e suporte financeiro da FAPESP.

${ }^{2}$ Pós-Graduação em Fitotecnia realizada na USP/ESALQ, Bolsista CNPq,
Programa DCR/Fundect, UFMS - Depto de Biologia, CP549, 79070-900, Campo Grande, MS, email: lianablima@gmail.com.

${ }^{3}$ USP/ESALQ - Depto de Produção Vegetal, CP09, 13418-900, Piracicaba, SP, Bolsista CNPq, email: jmarcos@esalq.usp.br. 


\section{INTRODUÇÃO}

O potencial fisiológico de lotes de sementes é avaliado pelo teste de germinação, realizado sob condições controladas e favoráveis. No entanto, quando as condições se afastam das ideais, no campo ou após um período de armazenamento, lotes com porcentagem de germinação semelhante podem apresentar desempenhos distintos devido a diferenças no vigor.

A Association of Official Seed Analysts (AOSA, 1983) conceitua vigor de sementes como a soma dos atributos determinantes do potencial para a emergência rápida e uniforme de plântulas normais sob ampla diversidade de condições ambientais. Assim, a estimativa do comportamento das sementes pode ser mais eficiente quando as informações sobre o vigor são obtidas pela combinação de testes, pois torna-se muito difícil que apenas um teste possa indicar, com certa precisão, o potencial de desempenho das sementes expostas a variadas situações (TeKrony, 2003; Marcos Filho, 2005).

Como consequência, a pesquisa sobre o vigor, visa dentre outros objetivos, o desenvolvimento de métodos simples, reproduzíveis, com rápida obtenção dos resultados, que permitam distinguir lotes de maior e de menor potencial fisiológico, cujos resultados estejam relacionados à emergência das plântulas em campo (Marcos Filho, 1999a).

Para sementes de grandes culturas, podem ser consultados na literatura inúmeros trabalhos que se referem ao estudo de testes para avaliação do vigor; entretanto é relativamente pequeno o volume de informações sobre procedimentos e as combinações mais adequadas para utilização de cada teste em sementes de pepino.

Há algumas indicações na literatura com relação a aplicação de testes de envelhecimento acelerado tradicional, $48 \mathrm{~h}$ de incubação a $41{ }^{\circ} \mathrm{C}$ (Bhéring et al., 2000) e 72 ou 96 horas a $41{ }^{\circ} \mathrm{C}$ (Torres, 2004) e para o procedimento com solução salina, $41^{\circ} \mathrm{C}$ por 72 horas (Abdo et al., 2005 e Bhéring et al., 2000), no entanto, não há um consenso sobre a combinação ideal.

Para o teste de deterioração controlada, os estudos realizados até o momento também originaram diferentes sugestões de metodologia, assim, Pandey et al. (1990) indicam o uso de sementes com $20 \%$ de teor de água a $40{ }^{\circ} \mathrm{C}$ por 48 ou 72 horas, enquanto, Bhéring et al. (2000) verificaram maior eficiência na distinção entre lotes quando utilizada a combinação $24 \%$ / 48 horas.

Desta maneira, o presente trabalho foi realizado com o objetivo de avaliar procedimentos para a condução de testes de vigor baseados na tolerância ao estresse térmico para avaliar o potencial fisiológico de sementes pepino.

\section{MATERIAL E MÉTODOS}

A pesquisa foi conduzida no Laboratório de Análise de Sementes da Escola Superior de Agricultura "Luiz de Queiroz" da Universidade de São Paulo (USP/ESALQ), em Piracicaba - SP, em três épocas de avaliação, com intervalos de 30 dias.

Utilizaram-se sete lotes de sementes de pepino, sendo quatro do cultivar Safira (produzidos pela empresa Sakata Seed Sudamerica) e três do cultivar Jóia (produzidos pela empresa Seminis Vegetable Seeds), com potenciais fisiológicos distintos. Os lotes foram homogeneizados periodicamente e permaneceram embalados em sacos de papel multifoliado e armazenados em câmara seca $\left(20^{\circ} \mathrm{C}\right.$ e $50 \%$ de umidade relativa do ar) durante o período de condução dos experimentos.

Os lotes foram avaliados através dos testes de germinação, emergência de plântulas, envelhecimento acelerado tradicional e com solução saturada de cloreto de sódio e deterioração controlada, determinando-se também o teor de água das sementes de cada lote. Os procedimentos utilizados estão descritos em seguida.

$\mathrm{O}$ teor de água foi determinado pelo método da estufa a $105 \pm 3{ }^{\circ} \mathrm{C}$, por 24 horas, com duas repetições de $1 \mathrm{~g}$ para cada tratamento (Brasil, 2009), os resultados foram expressos em porcentagem (base úmida).

Para o teste de germinação foram utilizadas quatro repetições de 50 sementes, distribuídas em rolos de papel toalha umedecidos com quantidade de água equivalente a 2,0 vezes a massa do substrato seco e colocada para germinar a $25{ }^{\circ} \mathrm{C}$. A contagem das plântulas normais foi realizada aos 4 e 8 dias após semeadura (Brasil, 2009) com resultados expressos em porcentagem média, para cada lote. A velocidade de germinação foi determinada durante a condução do teste, através de contagens diárias das plântulas normais até o oitavo dia após a semeadura. $O$ índice de velocidade de germinação foi obtido pela fórmula proposta por Maguire (1962), e os resultados foram expressos em índice médio de velocidade de germinação para cada lote.

No teste de emergência de plântulas também foram utilizadas quatro repetições de 50 sementes para cada lote. As sementes foram distribuídas em bandejas de poliestireno de 200 células contendo substrato Plantmax $®$. 
As bandejas foram mantidas em ambiente protegido, sob temperatura média de $25{ }^{\circ} \mathrm{C}$; no oitavo dia após a semeadura foram computadas as plântulas emergidas e os resultados foram expressos em porcentagem média para cada lote. A velocidade de emergência foi obtida de forma semelhante à de germinação, sendo computadas diariamente as plântulas emergidas até o oitavo dia após a semeadura, calculando-se o índice de velocidade de emergência de acordo com Maguire (1962).

O teste de envelhecimento acelerado foi conduzido de acordo com o método tradicional (utilização de 40 $\mathrm{mL}$ de água destilada como citado em AOSA, 1983) e o método com solução saturada de cloreto de sódio. Cada amostra de sementes (contendo em torno de 6 gramas) foi distribuída, formando camada única, sobre a superfície de tela metálica, suspensa no interior de caixa de plástico $(11 \times 11 \times 3 \mathrm{~cm})$, contendo $40 \mathrm{~mL}$ de água destilada (envelhecimento tradicional) ou $40 \mathrm{~mL}$ de solução saturada de cloreto de sódio (envelhecimento com solução saturada de sal). As caixas foram mantidas em câmara jaquetada de água a 41 e $45^{\circ} \mathrm{C}$, durante 48 , 72 e 96 horas. As temperaturas e períodos testados foram baseados nas combinações descritas na literatura para sementes de pepino (Bhéring et al., 2000; Torres, 2004; Abdo et al., 2005). Após o envelhecimento, foi conduzido teste de germinação computando-se a porcentagem de plântulas normais aos 4 dias após a semeadura.

Para o teste de deterioração controlada, o teor de água das sementes foi ajustado para 20 e $24 \%$ pelo método da atmosfera úmida, distribuindo-se as sementes formando uma camada única sobre a tela de alumínio suspensa no interior de caixa de plástico $(11 \times 11 \times 3 \mathrm{~cm})$ contendo 40 $\mathrm{mL}$ de água e mantida em germinador a $20{ }^{\circ} \mathrm{C}$ (Rossetto et al., 1995). Em seguida, as amostras foram mantidas em "banho-maria" a $45{ }^{\circ} \mathrm{C}$ (Alsadon et al., 1995) durante 24 e 48 horas. Após o término dos períodos, foi conduzido teste de germinação, computando-se a porcentagem de plântulas normais aos quatro dias após a semeadura. Os resultados foram expressos em porcentagem de plântulas normais para cada lote.

Os procedimentos para avaliação do potencial fisiológico foram repetidos em três épocas. Foi utilizado o delineamento inteiramente casualizado com quatro repetições separadamente para cada teste e cultivar; os dados foram submetidos à análise de variância e as médias foram comparadas pelo teste de Tukey $(\mathrm{p} \leq 0,05)$.

\section{RESULTADOS E DISCUSSÃO}

Os resultados obtidos nas três épocas de teste foram semelhantes, optando-se por apresentar apenas os resultados referentes à primeira época.

Os valores do teor de água e os resultados do teste de germinação e de emergência de plântulas são apresentados na Tabela 1. A análise de variância dos dados não acusou efeito de lotes sobre as porcentagens de germinação e de emergência de plântulas do cv. Safira, e porcentagem de germinação do cv. Jóia.

TABELA 1. Resultados da determinação do teor de água e dos testes de germinação e emergência de plântulas de lotes de sementes de pepino dos cultivares Safira e Jóia.

\begin{tabular}{|c|c|c|c|c|c|c|}
\hline \multirow{2}{*}{ Cultivar } & \multirow{2}{*}{ Lotes } & \multirow{2}{*}{$\begin{array}{c}\text { Teor de água } \\
\ldots . . . \% \ldots . . .\end{array}$} & \multicolumn{2}{|c|}{ Germinação } & \multicolumn{2}{|c|}{ Emergência de plântulas } \\
\hline & & & $\ldots . . \% \ldots$ & índice & $\ldots . . \% \ldots$ & Índice \\
\hline \multirow{5}{*}{ Safira } & 1 & 7,2 & $99 \mathrm{a}$ & $32,3 \mathrm{ab}$ & $99 \mathrm{a}$ & $69,7 \mathrm{a}$ \\
\hline & 2 & 6,9 & $99 \mathrm{a}$ & $33,1 \mathrm{a}$ & $100 \mathrm{a}$ & $68,8 \mathrm{a}$ \\
\hline & 3 & 7,1 & $100 \mathrm{a}$ & $32,8 \mathrm{ab}$ & $100 \mathrm{a}$ & $55,9 \mathrm{~b}$ \\
\hline & 4 & 7,2 & $96 \mathrm{a}$ & $31,6 \mathrm{~b}$ & $96 \mathrm{a}$ & $70,1 \mathrm{a}$ \\
\hline & C.V. $(\%)$ & - & 14,0 & 2,2 & 1,9 & 1,6 \\
\hline \multirow{4}{*}{ Jóia } & 5 & 7,8 & $99 \mathrm{a}$ & $33,3 \mathrm{a}$ & $99 \mathrm{a}$ & $70,7 \mathrm{a}$ \\
\hline & 6 & 7,6 & $96 a$ & $33,1 \mathrm{a}$ & $100 \mathrm{a}$ & $70,9 \mathrm{a}$ \\
\hline & 7 & 7,4 & $92 \mathrm{a}$ & $31,1 \mathrm{~b}$ & $89 \mathrm{~b}$ & $67,1 \mathrm{~b}$ \\
\hline & C.V. (\%) & - & 5,9 & 2,0 & 1,4 & 2,0 \\
\hline
\end{tabular}

Letras minúsculas: comparação das médias em cada coluna (Tukey a 5\% de probabilidade), separadamente para cada cultivar. 
O teor de água inicial das sementes dos lotes de ambos os cultivares variou entre 6,9 e $7,8 \%$; essa diferença está dentro dos limites considerados ideais em se tratando da aplicação de testes que avaliam o potencial fisiológico, não afetando o comportamento das sementes durante a condução dos testes e contribuindo para a obtenção de resultados consistentes (Marcos Filho, 1999b; TeKrony, 2003).

A porcentagem de germinação dos lotes do cv. Safira e do cv. Jóia foi semelhante, variando respectivamente, de 96 a $100 \%$ e 92 a $99 \%$. No entanto, verificou-se efeito de lotes para os índices de velocidade de germinação e de emergência de plântulas de ambas as cultivares, evidenciando a separação dos lotes em diferentes níveis de vigor. Assim, foi detectada a menor velocidade de germinação e de emergência dos lotes 3 e 4 do cv. Safira, respectivamente, e do lote 7 do cv. Jóia, mas não houve identificação nítida de um lote com melhor desempenho.

$\mathrm{Na}$ Tabela 2 podem ser observadas as médias do teor de água das sementes submetidas ao teste de envelhecimento acelerado tradicional e com solução saturada de sal, durante 48,72 e 96 horas, a 41 e $45^{\circ} \mathrm{C}$.

TABELA 2. Teor de água de lotes de sementes de pepino dos cultivares Safira e Jóia antes e após envelhecimento acelerado tradicional (EAT) e com solução saturada de sal (EASS), mantidas em câmara jaquetada por 48,72 e 96 horas, a 41 e $45^{\circ} \mathrm{C}$.

\begin{tabular}{|c|c|c|c|c|c|c|c|}
\hline \multirow{3}{*}{ Lotes } & \multirow{3}{*}{ Teor de água } & \multicolumn{6}{|c|}{ Teor de água após Envelhecimento acelerado a $41^{\circ} \mathrm{C}$} \\
\hline & & \multicolumn{2}{|c|}{48 horas } & \multicolumn{2}{|c|}{72 horas } & \multicolumn{2}{|c|}{96 horas } \\
\hline & & EAT & EASS & EAT & EASS & EAT & EASS \\
\hline & & \multicolumn{6}{|c|}{ 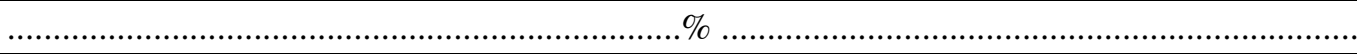 } \\
\hline 1 & 7,2 & 24,4 & 8,8 & 26,6 & 9,1 & 28,2 & 9,1 \\
\hline 2 & 6,9 & 23,4 & 8,5 & 22,6 & 9,0 & 25,9 & 10,1 \\
\hline 3 & 7,1 & 19,6 & 8,9 & 20,9 & 8,8 & 26,5 & 9,4 \\
\hline 4 & 7,2 & 25,6 & 9,6 & 23,7 & 9,8 & 28,2 & 9,1 \\
\hline 5 & 7,8 & 27,8 & 9,8 & 26,2 & 10,3 & 24,9 & 9,7 \\
\hline 6 & 7,6 & 25,0 & 9,8 & 26,9 & 9,8 & 20,5 & 9,4 \\
\hline \multirow[t]{2}{*}{7} & 7,4 & 25,5 & 9,2 & 22,7 & 9,8 & 22,0 & 10,3 \\
\hline & & \multicolumn{6}{|c|}{ Teor de água após Envelhecimento acelerado a $45^{\circ} \mathrm{C}$} \\
\hline \multirow[t]{3}{*}{ Lotes } & Teor de água & \multicolumn{2}{|c|}{48 horas } & \multicolumn{2}{|c|}{72 horas } & \multicolumn{2}{|c|}{96 horas } \\
\hline & & EAT & EASS & EAT & EASS & EAT & EASS \\
\hline & & \multicolumn{6}{|c|}{, } \\
\hline 1 & 6,8 & 24,9 & 10,6 & 27,8 & 10,8 & 26,8 & 10,1 \\
\hline 2 & 6,7 & 25,5 & 10,4 & 26,7 & 10,7 & 26,0 & 9,9 \\
\hline 3 & 6,5 & 23,9 & 9,8 & 25,5 & 10,0 & 25,7 & 9,5 \\
\hline 4 & 6,9 & 24,6 & 10,0 & 25,5 & 10,6 & 23,9 & 10,0 \\
\hline 5 & 7,1 & 23,2 & 10,1 & 25,2 & 10,5 & 23,8 & 9,6 \\
\hline 6 & 7,1 & 22,4 & 10,0 & 22,9 & 10,5 & 22,3 & 9,9 \\
\hline 7 & 7,0 & 22,4 & 9,9 & 24,4 & 10,4 & 22,5 & 10,0 \\
\hline
\end{tabular}

O teor de água inicial das sementes dos lotes e o verificado após o teste de envelhecimento acelerado com solução saturada de sal, a 41 e $45^{\circ} \mathrm{C}$, apresentou variação inferior ao limite tolerável de 2 pontos porcentuais sugerido na literatura, indicando uniformidade das condições do envelhecimento acelerado e contribuindo para a obtenção de resultados consistentes (Marcos Filho, 2005).

No entanto, quando foi utilizado o procedimento tradicional para o teste (EAT), as combinações $41{ }^{\circ} \mathrm{C} / 48$ h/72 h/96 h e $45^{\circ} \mathrm{C} / 72$ h/96 h testadas para o cv. Safira 
e as combinações $41{ }^{\circ} \mathrm{C} / 72 \mathrm{~h} / 96 \mathrm{~h}$ e $45{ }^{\circ} \mathrm{C} / 72 \mathrm{~h}$ testadas para o cv. Jóia originaram variações no teor de água superiores ao indicado como máximo tolerável. Essa variação, que leva a inconsistência nos resultados do teste de envelhecimento acelerado tradicional, pode ser decorrente de um efeito mais acentuado das condições da umidade relativa do ar elevada no comportamento das sementes. Quando são utilizadas soluções saturadas de sal, ao invés de água destilada, a umidade relativa do ar mantém-se mais baixa, levando a uma diminuição da velocidade de absorção de água pelas sementes e da intensidade de deterioração, com efeitos menos drásticos sobre as sementes e resultados menos variáveis (Jianhua e McDonald, 1996; Panobianco e Marcos Filho, 1998).

$\mathrm{Na}$ Tabela 3 estão os resultados do teste de envelhecimento acelerado tradicional e com solução saturada de sal em sementes mantidas em câmara jaquetada por 48,72 e 96 horas a 41 e $45^{\circ} \mathrm{C}$.

TABELA 3. Resultados do envelhecimento acelerado tradicional (EAT) e com solução saturada de sal (EASS) de lotes de sementes de pepino dos cultivares Safira e Jóia, mantidas em câmara jaquetada por 48, 72 e 96 horas, a 41 e $45^{\circ} \mathrm{C}$.

\begin{tabular}{|c|c|c|c|c|c|c|c|}
\hline \multirow{4}{*}{ Cultivar } & \multirow{4}{*}{ Lotes } & \multicolumn{6}{|c|}{$41^{\circ} \mathrm{C}$} \\
\hline & & \multicolumn{3}{|c|}{ EAT } & \multicolumn{3}{|c|}{ EASS } \\
\hline & & 48 horas & 72 horas & 96 horas & 48 horas & 72 horas & 96 horas \\
\hline & & . & $\ldots$ & . & ………........ & ................... & $\ldots$ \\
\hline \multirow{5}{*}{ Safira } & 1 & $94 \mathrm{~b}$ & $96 \mathrm{a}$ & $91 \mathrm{~b}$ & $97 \mathrm{a}$ & $97 \mathrm{a}$ & $96 \mathrm{ab}$ \\
\hline & 2 & $100 \mathrm{a}$ & $99 \mathrm{a}$ & $100 \mathrm{a}$ & $98 \mathrm{a}$ & $99 \mathrm{a}$ & $98 \mathrm{a}$ \\
\hline & 3 & $99 \mathrm{a}$ & 97 a & $98 \mathrm{a}$ & 98 a & $97 \mathrm{a}$ & $92 \mathrm{~b}$ \\
\hline & 4 & $89 \mathrm{c}$ & $84 \mathrm{~b}$ & $81 \mathrm{c}$ & $94 \mathrm{a}$ & $95 \mathrm{a}$ & $93 \mathrm{ab}$ \\
\hline & C.V. (\%) & 2,3 & 3,2 & 2,8 & 2,7 & 2,6 & 3,1 \\
\hline \multirow{4}{*}{ Jóia } & 5 & $100 \mathrm{a}$ & $98 \mathrm{a}$ & 99 a & $98 \mathrm{a}$ & $95 \mathrm{a}$ & $93 \mathrm{a}$ \\
\hline & 6 & $97 \mathrm{a}$ & $96 \mathrm{a}$ & $96 \mathrm{ab}$ & $91 \mathrm{ab}$ & $90 \mathrm{ab}$ & $86 a b$ \\
\hline & 7 & $93 \mathrm{~b}$ & $90 \mathrm{~b}$ & $92 \mathrm{~b}$ & $79 \mathrm{~b}$ & $82 \mathrm{~b}$ & $82 \mathrm{~b}$ \\
\hline & C.V. (\%) & 1,2 & 2,7 & 2,9 & 6,9 & 6,1 & 4,9 \\
\hline \multirow{4}{*}{ Cultivar } & \multirow{4}{*}{ Lotes } & \multicolumn{6}{|c|}{$45^{\circ} \mathrm{C}$} \\
\hline & & \multicolumn{3}{|c|}{ EAT } & \multicolumn{3}{|c|}{ EASS } \\
\hline & & 48 horas & 72 horas & 96 horas & 48 horas & 72 horas & 96 horas \\
\hline & & \multicolumn{6}{|c|}{ 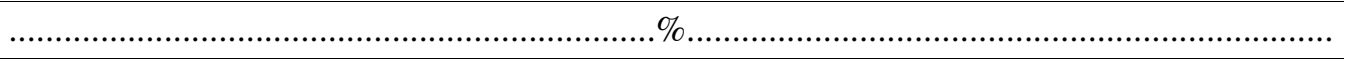 } \\
\hline \multirow{5}{*}{ Safira } & 1 & $93 \mathrm{a}$ & $87 \mathrm{~b}$ & $78 \mathrm{a}$ & $92 \mathrm{a}$ & $96 \mathrm{a}$ & $92 \mathrm{a}$ \\
\hline & 2 & $98 \mathrm{a}$ & $96 \mathrm{a}$ & $95 \mathrm{a}$ & $94 \mathrm{a}$ & $91 \mathrm{a}$ & $90 \mathrm{a}$ \\
\hline & 3 & 99 a & 96 a & $88 \mathrm{a}$ & 96 a & $80 \mathrm{~b}$ & $85 \mathrm{a}$ \\
\hline & 4 & $91 \mathrm{a}$ & $73 \mathrm{c}$ & $50 \mathrm{~b}$ & $94 \mathrm{a}$ & $90 \mathrm{a}$ & $91 \mathrm{a}$ \\
\hline & C.V. $(\%)$ & 4,6 & 3,9 & 10,4 & 4,2 & 4,5 & 4,4 \\
\hline \multirow{4}{*}{ Jóia } & 5 & $96 \mathrm{a}$ & $97 \mathrm{a}$ & $92 \mathrm{a}$ & $92 \mathrm{a}$ & $82 \mathrm{a}$ & $88 \mathrm{a}$ \\
\hline & 6 & $96 \mathrm{a}$ & $90 \mathrm{ab}$ & $81 \mathrm{a}$ & $81 \mathrm{ab}$ & $79 a$ & $84 a b$ \\
\hline & 7 & $89 \mathrm{a}$ & $82 \mathrm{~b}$ & $63 \mathrm{~b}$ & $78 \mathrm{~b}$ & $78 \mathrm{a}$ & $77 \mathrm{~b}$ \\
\hline & C.V. (\%) & 7,4 & 7,3 & 7,9 & 7,4 & 6,9 & 6,9 \\
\hline
\end{tabular}

Letras minúsculas: comparação de médias em cada coluna (Tukey a 5\% de probabilidade), separadamente para cada cultivar. 
Para os lotes do cv. Safira, algumas combinações testadas foram eficientes para separar os lotes em diferentes níveis de vigor, de maneira condizente com os testes iniciais (Tabela 1). Assim, o envelhecimento acelerado tradicional a $41{ }^{\circ} \mathrm{C}$ indicou o lote 4 como o de menor vigor e os lotes 2 e 3 , de maior vigor, havendo separação mais nítida dos lotes quando utilizado o período de 96 horas. O envelhecimento acelerado com solução saturada de sal permitiu separação entre os lotes somente quando foi utilizado o período de 96 horas. Nessa combinação, o teste indicou o lote 3 como o de vigor inferior e destacou a superioridade do lote 2 .

Já nos lotes do cv. Jóia, o uso do procedimento tradicional e com solução saturada de sal a $41{ }^{\circ} \mathrm{C}$, foi eficiente em detectar diferenças de vigor entre os lotes, indicando o lote 7 como o de menor vigor e o lote 5 de maior vigor. Entretanto, no procedimento tradicional, o teor de água verificado após $72 \mathrm{~h}$ a 41 e $45^{\circ} \mathrm{C}$ e $96 \mathrm{~h}$ a 41 ${ }^{\circ} \mathrm{C}$, excederam o limite tolerável de 2 pontos percentuais de diferença entre os lotes avaliados.

Com o uso da temperatura $45{ }^{\circ} \mathrm{C}$ e período de 96 horas (tabela 3) para o cv. Safira, os resultados do envelhecimento acelerado com solução saturada de sal não foram condizentes com os demais testes; da mesma maneira ocorreu no envelhecimento acelerado tradicional, com 48 horas de envelhecimento (Tabela 1).

De modo geral, para o teste de envelhecimento acelerado, os procedimentos mais promissores para avaliação do potencial fisiológico de sementes, foram o envelhecimento conforme o procedimento tradicional ou com solução saturada de sal, durante 96 horas em câmara jaquetada a $41{ }^{\circ} \mathrm{C}$. É importante salientar que o procedimento tradicional a 41 ou $45^{\circ} \mathrm{C}$ originaram maiores diferenças nos teores de água entre os lotes testados, o que pode comprometer resultados posteriores ou tornar o teste mais longo, devido à possível necessidade de repetição das avaliações na hipótese de obtenção de teores de água finais acima dos toleráveis. Abdo et al. (2005) também relataram para sementes de pepino a maior variação no teor de água das sementes quando utilizado o procedimento tradicional.

Os efeitos do envelhecimento acelerado com solução saturada de cloreto de sódio, em substituição à água, de modo geral, foram menos drásticos, mas ao mesmo tempo houve menor sensibilidade para a deteç̧ão de diferenças de vigor entre os lotes de sementes. Além disso, a uniformidade do teste avaliada pelo teor de água final foi mais consistente do que com o uso do procedimento tradicional. Esse comportamento foi observado também por Jianhua e McDonald (1996) e Panobianco e Marcos Filho (2001).

As informações obtidas confirmaram a possibilidade da utilização do teste de envelhecimento acelerado tradicional ou com solução saturada de sal a $41{ }^{\circ} \mathrm{C}$ por 96 horas, para detectar diferenças no potencial fisiológico de sementes de pepino. Esse resultado concorda com os obtidos por Torres (2005a) também em sementes de pepino, e por Torres e Marcos Filho (2003) em sementes de melão. No entanto, difere dos obtidos por Abdo et al. (2005) e Bhering et al. (2000), que concluíram que o procedimento com solução saturada de sal a $41{ }^{\circ} \mathrm{C}$ por 72 horas foi o mais eficiente para sementes de pepino.

Como indicam os resultados verificados na literatura para sementes de pepino, a combinação dos fatores temperatura e período de exposição no teste de envelhecimento acelerado, por exemplo, pode ser adequada para determinado conjunto de lotes utilizado em um trabalho de pesquisa, porém, pode não ser para outro. Se os lotes utilizados em determinada pesquisa apresentarem potencial fisiológico elevado e semelhante, podem não ser diferenciados quanto ao vigor quando submetidos a períodos menores de envelhecimento. Da mesma forma, um período mais longo pode ser muito drástico para um conjunto de lotes de potencial menor e então, não ser eficiente para distingui-los em diferentes níveis de vigor. Assim, a definição de um procedimento eficiente para avaliar o vigor de sementes de determinada espécie não é tarefa fácil, pois é necessário, dentre outros, avaliar um número grande de lotes de variados cultivares.

$\mathrm{Na}$ Tabela 4 estão os dados médios referentes ao teor de água antes e após o ajuste para 20 e $24 \%$, colocadas em banhomaria a $45{ }^{\circ} \mathrm{C}$, para realização da deterioração controlada por 24 e 48 horas.

O teor de água inicial das sementes dos diferentes lotes, de cada cultivar, não apresentou variação elevada, o que é importante, pois essa uniformidade é essencial para a padronização das avaliações e obtenção de resultados consistentes (Marcos Filho, 1999a, 2001).

Essa pequena variação, também foi verificada após os ajustes do teor de água para atingir os valores de 20 e 24\%; assim, esse procedimento foi eficiente, pois os valores foram uniformes entre os lotes e muito próximos aos planejados. TeKrony (2003) alertou que alguns resultados revelam que um ponto porcentual de diferença no teor de água pode causar grande impacto na germinação após a deterioração controlada, principalmente para os lotes de médio e baixo vigor. Dessa forma, o teste exige um maior 
treinamento técnico para que possa ser conduzido com sucesso (Powell e Matthews, 1981).

$\mathrm{Na}$ Tabela 5 estão apresentados os resultados referentes ao teste de deterioração controlada. Algumas combinações foram eficientes para separar os lotes em níveis de vigor.

TABELA 4. Dados médios referentes ao teor de água de sementes de pepino dos cultivares Safira e Jóia ajustados para 20 e $24 \%$, antes e após deterioração controlada por 24 e 48 horas.

\begin{tabular}{|c|c|c|c|c|c|c|}
\hline \multirow{3}{*}{ Cultivar } & \multirow{3}{*}{ Lotes } & \multirow{2}{*}{ Teor de água } & \multicolumn{2}{|c|}{24 horas } & \multicolumn{2}{|c|}{48 horas } \\
\hline & & & $20 \%$ & $24 \%$ & $20 \%$ & $24 \%$ \\
\hline & & 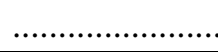 & $\ldots+\ldots$ & $\% \ldots .$. & $\ldots \ldots \ldots . .$. & ............ \\
\hline \multirow{4}{*}{ Safira } & 1 & 6,8 & 20,8 & 23,6 & 19,7 & 23,7 \\
\hline & 2 & 6,7 & 20,7 & 24,5 & 19,9 & 23,7 \\
\hline & 3 & 6,5 & 20,0 & 23,9 & 20,0 & 23,9 \\
\hline & 4 & 6,9 & 20,8 & 24,0 & 19,5 & 23,7 \\
\hline \multirow{3}{*}{ Jóia } & 5 & 7,1 & 20,6 & 23,6 & 19,7 & 23,9 \\
\hline & 6 & 7,1 & 19,8 & 23,8 & 19,9 & 23,5 \\
\hline & 7 & 7,0 & 19,7 & 24,5 & 19,8 & 23,6 \\
\hline
\end{tabular}

TABELA 5. Resultados de deterioração controlada de lotes de sementes de pepino dos cultivares Safira e Jóia realizada em sementes com teores de água ajustados para 20 e $24 \%$, por períodos de 24 e 48 horas, avaliada no quarto dia após semeadura.

\begin{tabular}{|c|c|c|c|c|c|}
\hline \multirow{3}{*}{ Cultivar } & \multirow{3}{*}{ Lotes } & \multicolumn{2}{|c|}{24 horas } & \multicolumn{2}{|c|}{48 horas } \\
\hline & & $20 \%$ & $24 \%$ & $20 \%$ & $24 \%$ \\
\hline & & \multicolumn{4}{|c|}{ … } \\
\hline \multirow{5}{*}{ Safira } & 1 & $99 \mathrm{a}$ & $97 \mathrm{a}$ & $93 \mathrm{bc}$ & $95 \mathrm{a}$ \\
\hline & 2 & 99 a & 99 a & $97 \mathrm{ab}$ & 98 a \\
\hline & 3 & 99 a & $100 \mathrm{a}$ & 98 a & $94 \mathrm{ab}$ \\
\hline & 4 & $96 \mathrm{a}$ & 96 a & $92 \mathrm{c}$ & $88 \mathrm{~b}$ \\
\hline & C.V. (\%) & 1,5 & 2,5 & 2,1 & 3,4 \\
\hline \multirow{4}{*}{ Jóia } & 5 & $99 \mathrm{a}$ & $99 \mathrm{a}$ & $100 \mathrm{a}$ & $97 \mathrm{a}$ \\
\hline & 6 & $97 \mathrm{a}$ & $98 \mathrm{a}$ & $95 \mathrm{a}$ & $90 \mathrm{ab}$ \\
\hline & 7 & $93 \mathrm{~b}$ & $93 \mathrm{~b}$ & $86 \mathrm{~b}$ & $84 \mathrm{~b}$ \\
\hline & C.V.(\%) & 1,8 & 2,2 & 3,6 & 7,2 \\
\hline
\end{tabular}

Letras minúsculas: comparação de médias em cada coluna (Tukey a 5\% de probabilidade), separadamente para cada cultivar.

De modo geral, para os cultivares avaliados, o período de 24 horas em banho-maria não foi suficiente para provocar separação consistente entre os lotes. A utilização do período de 48 horas foi mais sensível para detectar diferenças no vigor, tanto com o ajuste do teor de água para $20 \%$ como para $24 \%$.

Para o cv. Safira, os resultados revelaram o lote 4 como o de menor vigor, não havendo nítida distinção entre os demais quanto ao lote de maior vigor.

Para os lotes do cv. Jóia, de modo geral, o ajuste do teor de água para 20 e $24 \%$ em combinação com o período de 48 horas foram eficientes para indicar o lote 7 como o de menor vigor e o lote 5 o de maior, resultado semelhante ao verificado nos testes iniciais (Tabela 1) e no teste de envelhecimento acelerado.

De modo geral, a combinação entre o ajuste do teor 
de água das sementes para 20 ou $24 \%$ e período de 48 horas em banho-maria, a $45{ }^{\circ} \mathrm{C}$ demonstrou eficiência na avaliação do vigor de sementes pelo teste de deterioração controlada.

A proposta inicial para o teste de deterioração controlada (Matthews, 1980) sugere que o ajuste no teor de água seja tal que o período de 24 horas permita a separação entre os lotes de sementes pequenas. Assim, Panobianco e Marcos Filho (1998) e Torres (2005b) obtiveram resultados favoráveis com o uso da deterioração controlada, respectivamente, em sementes de pimentão e de maxixe com $24 \%$ de teor de água, a $45^{\circ} \mathrm{C}$ por 24 horas.

Entretanto, Bhering et al. (2004) verificaram para sementes de melão que a deterioração pode ser realizada por 48 horas a $45{ }^{\circ} \mathrm{C}$ em sementes com $24 \%$ de teor de água. Já para sementes de melancia, Bhering et al. (2001) verificaram que o teste de deterioração controlada foi mais adequado quando utilizaram temperatura mais baixa, 41 ${ }^{\circ} \mathrm{C}$, no mesmo período teor de água.

Para sementes de pepino, os estudos realizados até o momento originaram diferentes sugestões de metodologia. Assim, Alsadon et al. (1995) avaliaram sementes com $24 \%$ de água colocadas em banho-maria a $45{ }^{\circ} \mathrm{C}$, por 6 , $18,24,30,42,48$ e 72 horas, e verificaram que nenhum dos períodos testados foi eficiente para separar os lotes testados. Os resultados de Pandey et al. (1990) indicam o uso de sementes com $20 \%$ de teor de água a $40{ }^{\circ} \mathrm{C}$ por 48 ou 72 horas. Bhering et al. (2000) avaliaram o uso de $24 \mathrm{e}$ 48 horas de banho-maria a $45^{\circ} \mathrm{C}$ em sementes com teor de água ajustado para 20 e 24\%, e verificaram maior eficiência na distinção entre lotes quando utilizada a combinação $24 \%$ de água e 48 horas em banho-maria.

Um aspecto importante em uma rotina onde se utiliza o teste de deterioração controlada é a precisão requerida no ajuste do teor de água das sementes (Powell e Matthews, 1981), no entanto esse ajuste requer determinado período de tempo para ser realizado e é uma etapa trabalhosa. Muniz et al. (2004) obtiveram resultados satisfatórios na avaliação do potencial fisiológico de sementes de melão pelo teste de deterioração controlada, porém, relatou que a aplicação do teste foi trabalhosa e considerou esse fato uma desvantagem em relação ao teste de envelhecimento acelerado. Powell e Matthews (1981) também relataram que o teste de deterioração controlada requer maior treinamento técnico para sua aplicação do que o de envelhecimento acelerado.

Por meio da análise dos resultados foi possível identificar testes eficientes para avaliar o vigor de sementes de pepino. Os resultados fornecidos pelo teste de envelhecimento acelerado e de deterioração controlada foram consistentes e permitiram separação dos lotes em diferentes níveis de vigor.

\section{CONCLUSÃO}

Os testes de envelhecimento acelerado $\left(41{ }^{\circ} \mathrm{C}\right.$ por 96 horas nos procedimentos tradicional e com solução saturada de sal) e deterioração controlada (teor de água ajustado para $24 \%, 48$ horas em banho-maria a $45^{\circ} \mathrm{C}$ ) são eficientes para avaliar o vigor de sementes de pepino.

\section{REFERÊNCIAS}

ABDO, M.T.V.N.; PIMENTA, R.S.; PANOBIANCO, M.; VIEIRA, R.D. Testes de vigor para avaliação de sementes de pepino. Revista Brasileira de Sementes, v.27, n.1, p.195-198, 2005.

ALSADON, A.; YULE, L.J.; POWELL, A.A. Influence of seed ageing on the germination, vigour and emergence in module trays of tomato and cucumber seeds. Seed Science and Technology, v.23, p.665-672, 1995.

ASSOCIATION OF OFFICIAL SEED ANALYSTS AOSA. Seed vigor testing handbook. AOSA, Wageningen, 1983. 88p. (Handbook on seed testing. Contribuition, 32).

BHERING, M.C.; DIAS, D.C.F.S.; GOMES, J.M.; BARROS, D.I. Métodos para avaliação do vigor de sementes de pepino. Revista Brasileira de Sementes, v.22, n.1, p.171-175, 2000.

BHERING, M.C.; BARROS, D.I.; DIAS, D.C.F.S. Avaliação do vigor de sementes de melancia pelos testes de envelhecimento acelerado e deterioração controlada. Informativo ABRATES, v.11, n.2, p.204, 2001.

BHERING, M.C.; DIAS, D.C.F.S.; TOKUHISA, D.; DIAS, L.A. dos S. Avaliação do vigor de sementes de melão pelo teste de deterioração controlada. Revista Brasileira de Sementes, v.26, n.1, p.125-129, 2004.

BRASIL. Ministério da Agricultura, Pecuária e Abastecimento. Regras para análise de sementes. Ministério da Agricultura, Pecuária e Abastecimento. Secretaria de Defesa Agropecuária. Brasília, DF: Mapa/ ACS, 2009. 395p.

JIANHUA, Z.; MCDONALD, M.B. The saturated salt accelerated aging test for small seeded crops. Seed Science and Technology, v.25, n.1, p.123-131, 1996.

MAGUIRE, J.D. Speed of germination-aid in relation 
evaluation for seedling emergence vigor. Crop Science, v.2, n.2, p.176-177, 1962.

MARCOS FILHO, J. Testes de vigor: importância e utilização. In: KRZYZANOWSKI, F.C.; VIEIRA, R.D.; FRANÇA NETO, J.de B. (Ed.). Vigor de sementes: conceitos e testes. Londrina: Londrina: Associação Brasileira de Tecnologia de Sementes, Comitê de vigor de sementes, cap. 1, p. 1-20, 1999a.

MARCOS FILHO, J. Testes de envelhecimento acelerado. In: KRZYZANOWSKI, F.C.; VIEIRA, R.D.; FRANÇA NETO, J.de B. (Ed.). Vigor de sementes: conceitos e testes. Londrina: ABRATES, cap.3, p.1-24, 1999b.

MARCOS FILHO, J. Pesquisa sobre vigor de sementes de hortaliças. Informativo ABRATES, v.11, n.3, 2001, p.6375.

MARCOS FILHO, J. Fisiologia de sementes de plantas cultivadas. Piracicaba: Fealq, 2005, 495p.

MATTHEWS, S. Controlled deterioration: a new vigour test for crop seeds. In: HABBLETHWAITE, P.D. (Ed.). Seed Production. London: Butterworths, 1980. p.647660.

MUNIZ, M.F.B.; GONÇALVES, N.; GARCIA, D.C.; KULCZYNSKI, S.M. Comparação entre métodos para avaliação da qualidade fisiológica e sanitária de sementes de melão. Revista Brasileira de Sementes, v.26, n.2, p.144-149, 2004.

PANDEY,P.K.; GOYAL, R.D.; PRAKASH, V.; JATIVER, R.P.; SINGH. Association between laboratory vigour tests and fields emergence in cucurbits. Seed Research, v.18, p.40-43, 1990.
PANOBIANCO, M.; MARCOS FILHO, J. Comparação entre métodos para avaliação da qualidade de sementes de pimentão. Revista Brasileira de Sementes, v.20, n.2, p.306-310, 1998.

PANOBIANCO, M.; MARCOS FILHO, J. Envelhecimento acelerado e deterioração controlada em sementes de tomate. Scientia Agricola, v.58, n.3, p.525-531, 2001.

POWELL, A.A.; MATTHEWS, S. Evaluation of controlled deterioration, a new vigour test for small seed vegetables. Seed Science and Technology, v.9, p.633-640, 1981.

ROSSETTO, C.A.V.; FERNANDEZ, E.M.; MARCOS FILHO, J. Metodologias de ajuste do grau de umidade e comportamento das sementes de soja no teste de germinação. Revista Brasileira de Sementes, v.17, n.2, p.171-178, 1995.

TeKRONY, D.M. Precision is an essential component in seed vigour testing. Seed Science and Technology, v.31, p.435-447, 2003.

TORRES, S.B. Envelhecimento acelerado em sementes de pepino. Horticultura Brasileira, Brasília, v. 22, n. 2, 2004 (suplemento) 1 CD-ROM.

TORRES, S.B. Envelhecimento acelerado em sementes de pepino com e sem solução salina saturada. Horticultura Brasileira, v.23, n.2, p.303-306, 2005a.

TORRES, S.B. Teste de deterioração controlada em sementes de maxixe. Horticultura Brasileira, v. 23, n. 2, p.307-310, 2005b.

TORRES, S.B.; MARCOS FILHO, J. Accelerated aging of melon seeds. Scientia Agricola, v.60, n.1, p.77-82, 2003. 